\title{
A Study on Factors Influencing University Students' Willingness to Continue Using Mobile Learning in the Post-Coronavirus Period
}

\author{
Jiuhong Yin $^{1 *}$ and Jinghuai She ${ }^{2}$ \\ ${ }^{1}$ Suzhou Institute of Industrial Technology Suzhou, Jiangsu, China \\ ${ }^{2}$ Capital University of Economics and Business Beijing, China
}

\begin{abstract}
Mobile learning exposes some problems during the process of fast development. As the epidemic gradually stabilized, the willingness to continue using mobile learning began to decline. In order to improve the learning effect of mobile learning users and increase the public's recognition of the form of mobile learning, this article takes university students, one of the main users of mobile learning, as the research object, explores the factors that affect the willingness of university students to continue using mobile learning, and further introduces self-efficacy, perceive variables such as teacher support, and investigate in depth the influence mechanism of mobile learning users' self-perception and online teacher service perception on the willingness to continue using mobile learning. This study has found that compared with perceived ease of use, mobile learning can improve satisfaction, perceived usefulness, and perceived teacher support and self-efficacy services that can more significantly enhance students' willingness to continue using mobile learning.
\end{abstract}

\section{Introduction}

With the continuous development of 5G technology and mobile devices, mobile learning has become an important method and tool for Chinese students' daily learning. Mobile learning is of great significance to realize and accelerate the construction of a learning society where everyone can learn everywhere and anytime. Therefore, based on the theory of technology acceptance and combining the characteristics of university students' mobile learning, this article constructs a model of the factors affecting the continued use of university students' mobile learning willingness. Through empirical research and design, explore ways to improve the quality and effect satisfaction of university students' mobile learning. It is expected to provide a basis for educational institutions and other departments to formulate corresponding learning, guidance and support strategies according to their own needs, promote the popular use of mobile learning methods, and help my country build a learning society.

* Corresponding author: anna9919@126.com 


\section{Theories and Hypothesis}

In recent years, more and more attention has been paid to the research of continuous behavior of mobile learning. In order to make mobile learning more beneficial to mobile learners and achieve better learning results, it is necessary to understand the factors that affect students' continuous acceptance of mobile learning, so as to better implement mobile learning. Research can be divided into two aspects: on the one hand, research on the influencing factors of mobile learning. Based on one or more of the theories of planned behavior theory (TPB is short for "theory of planned behaviour", the same goes for the following), technology acceptance model theory (TAM) and integrated technology acceptance theory (UTAUT), it was confirmed that the factors influencing students' intentions to apply mobile learning were perceived usefulness, perceived easiness to employ, attitude, self-efficacy and performance expectation. [1-3] On the other hand, combined with the study of university students' mobile learning conditions, students' readiness, self-efficacy in using mobile devices and personal innovation have an impact on the effect of mobile learning, [4] but the most influential acceptance factors of mobile learning are "general ability" and "learning activities".

Based on the relationship of variables in TAM theory, this paper puts forward the following hypotheses:

H1: Perceived ease of use has a positive impact on perceived usefulness.

H2: Perceived ease of use has a positive impact on satisfaction.

Although the domestic literature on the impact of mobile learning acceptance intention have laid a foundation for our research, they also have the following limitations: Firstly, the key influencing factors of mobile learning are studied from a single level or a combination of several levels, such as individuals, technology, task or environment respectively, while the influence of teacher support on university students' acceptance intention of mobile learning is almost not involved. In fact, the learning environment supported by teachers can stimulate students' internal motivation and initiative for learning. [5] In the process of mobile learning, students' perception of teachers' supportive behavior and attitude will improve their overall perception of all aspects of their own learning, that is, self-efficacy. The study found that students' perception of teachers' support from all aspects had an impact on their behavior and learning. When students perceive more supportive behaviors of teachers, such as care, encouragement, guidance and help, their learning enthusiasm and learning confidence will be stronger, which stimulate students' interest in learning, and the learning quality and learning effect may be improved. In this process, it is not difficult to see that the perceived teacher support provides guidance and help for students, which on the one hand guarantees the quality of mobile learning for students; on the other hand, enables students to receive incentives and care, and thus they will generate more positive comments on mobile learning. Therefore, this paper proposes the following hypotheses:

H3: Perceived teacher support has a positive impact on satisfaction with mobile learning.

H4: Perceived teacher support has a positive impact on perceived usefulness.

H5: Perceived teacher support has a positive impact on self-efficacy.

Self-efficacy of mobile learning is the product of interaction among learning behavior, individual factors and external environment. It affects learners' perception of mobile learning and their control of learning behavior. The stronger the self-efficacy is, the more confident the learner is to gain in the mobile learning activities, and the more easily he or she can control his or her learning actions. In this case, the more students perceive the use of mobile learning, the better the effect of mobile learning will be. Therefore, this paper proposes the following hypotheses:

H6: Self-efficacy of mobile learning has a positive impact on perceived usefulness.

H7: Self-efficacy of mobile learning has a positive impact on perceived ease of use 
According to Bhattacherjee's model of continuous use of information systems, perceived usefulness has a significant impact on the intention to continue using information systems. [6] Therefore, the perceived usefulness in this study refers to the degree to which university students can improve their learning efficiency or quality after using mobile learning. Based on this model, it is also found that when students can better and more efficiently acquire required resources and complete learning tasks after mobile learning, users will be satisfied with using mobile learning, namely, perceived usefulness will affect satisfaction. Therefore, this paper proposes the following hypotheses:

H8: Perceived usefulness has a positive impact on the willingness to continue using mobile learning.

H9: Satisfaction has a positive impact on the willingness to continue using mobile learning.

H10: Perceived usefulness has a positive impact on satisfaction.

To sum up, the theoretical model established in figure1.

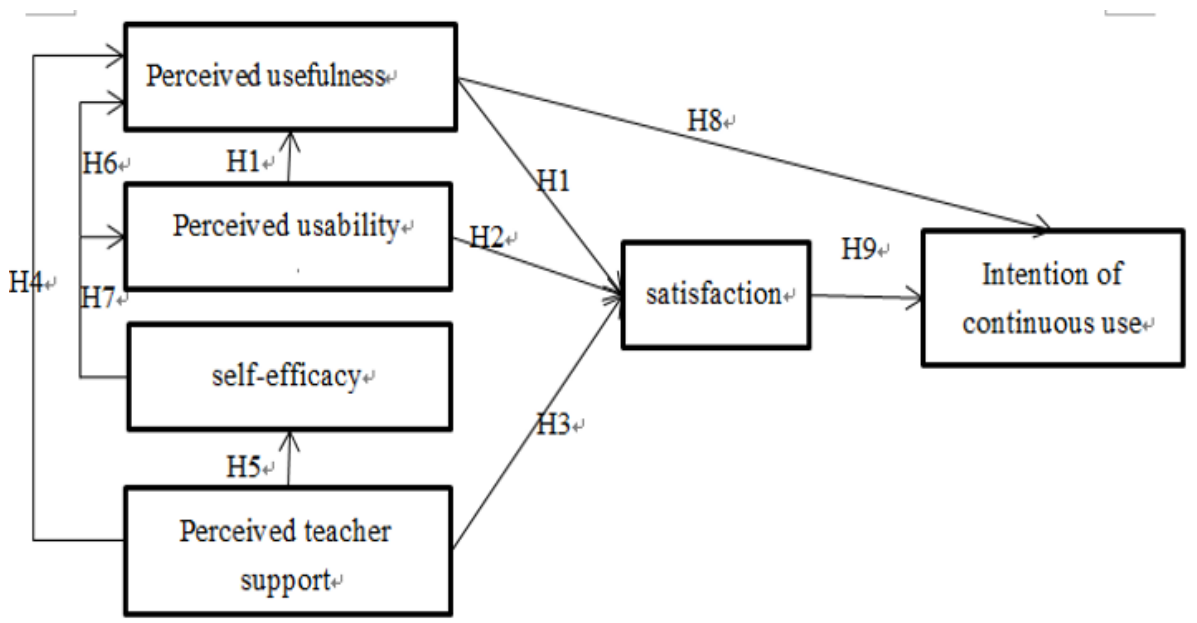

Figure 1 model of university students' willingness to continue using mobile learning

\section{Questionnaire design and data collection}

Questionnaires were designed according to the influencing factors model of university students' willingness to continue using mobile learning constructed above. In order to ensure that the questionnaire has sufficient reliability and validity. On the basis of previous studies on the maturity scale and combining with the characteristics of the research objects, this study modified and improved some questions. The questionnaire used a Likert 7-point scale, and the respondents answered the questions according to the perceptual experience of using mobile learning. After preliminary investigation and modification, some questions with low reliability and validity were removed, and 6 dimensions and 25 questions were formed. The six dimensions are directed to continue using, satisfaction, perceived usefulness, perceived ease of use, self-efficacy, and perceived teacher support.

Through online distribution and collection, 684 questionnaires were collected. Among the respondents, $66.7 \%$ were women and 33.3\% men. After removing 102 samples that have never had mobile learning experience and 102 invalid samples (such as choosing the same answer to all questions), there were 582 valid questionnaires, with the response rate $85 \%$. 


\section{Empirical analysis}

In this paper, the reliability analysis is carried out by using standardized factor load, topic reliability, combination reliability and Cronbach's alpha. SMC (also known as R2) reflects the extent to which individual measurement variables are affected by potential variables. If the standardized coefficient load is greater than 0.6, the SMC is greater than 0.5 , the combined reliability CR is greater than 0.8 , and the AVE is greater than 0.5 , the reliability and validity of the item are acceptable. Through the reliability analysis of the questionnaire, except Q11 and Q28, all items passed the above reliability index test. The results show that the overall reliability of the questionnaire is good and reliable. The above data show that the questionnaire has a good measurement reliability.

The validity of the measurement was mainly tested by convergence validity and differentiation validity. The convergence validity is the factor load value and the square difference extraction value (AVE). The discriminant validity is realized by calculating the difference between the coefficient correlation between the variables and the square root of the square deviation extracted value of the corresponding variables. The square root of average value is higher than the correlation coefficient between variables, which indicates that the questionnaire has a good validity. The convergence validity value Ave in the table above is basically greater than 0.5 , so it is judged that the convergence validity of the data in this paper is good.

In this paper, 5 of the most frequently used indicators were selected to evaluate the model fitness. CMIN/DF, RMSEA, GFI, AGFI and TLI in this study are all within the acceptable range, indicating that the research model basically meets the requirements and has good adaptability

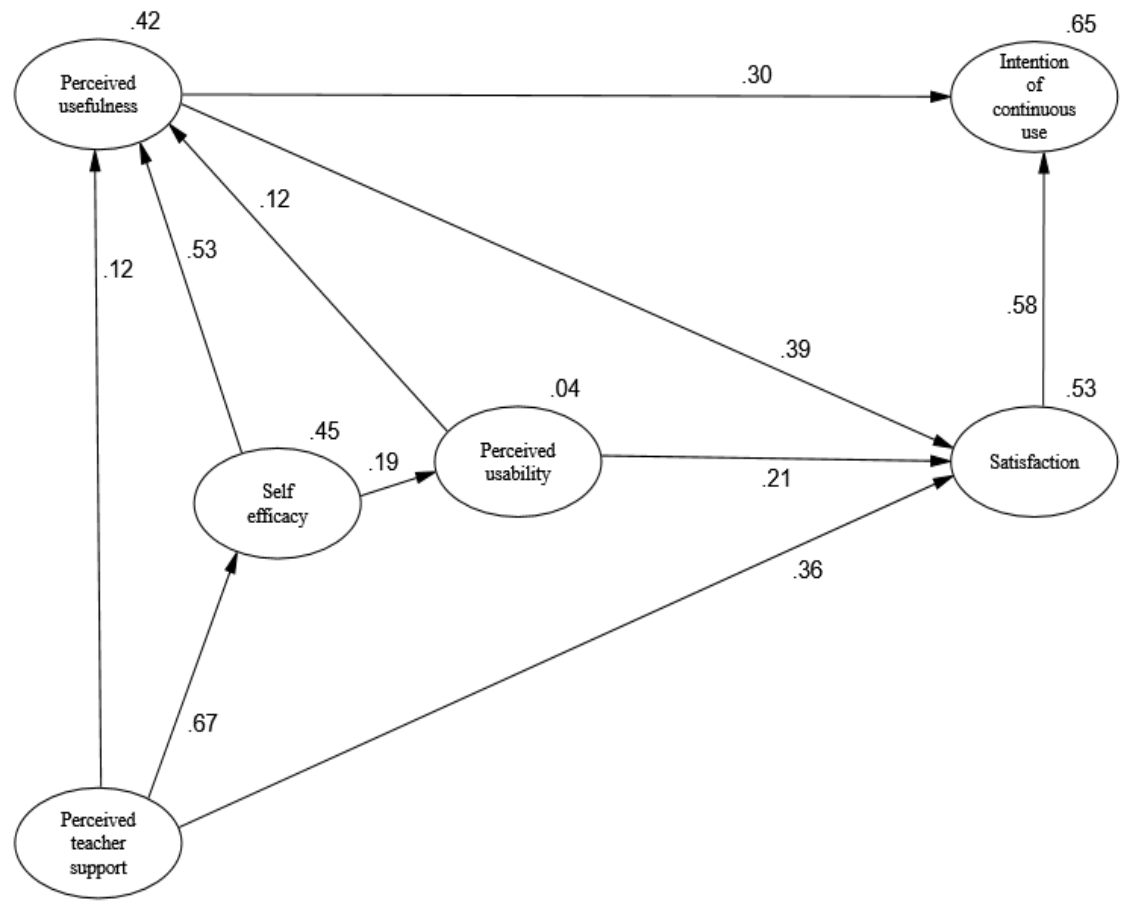

Figure 2 Model normalization path coefficient 
This study examined the significance of the relationship between variables according to the critical ratio and $\mathrm{P}$ value. When the critical ratio is greater than 1.95 and $\mathrm{P}$ is less than 0.05 , the path coefficient is significant. Table 6.6 shows the test results of each path. It can be seen that the 10 paths proposed in this paper are all significantly valid. In conclusion, all the 10 hypotheses proposed in this study have been proved. The size of each path coefficient can be seen in the figure2.

In order to study the differences among the influencing factors of university students' continuous use of mobile learning and analyze the direct or indirect effects of variables on their intention to continue using, this paper conducts the following path analysis to explore the mechanism of action among variables.

\section{Summary and Discussion}

According to the results of path analysis, in order of the total effect, satisfaction (0.580), perceived usefulness $(0.531)$, perceived teacher support $(0.486)$, self-efficacy $(0.316)$, and perceived ease of use (0.184) were ranked in descending order. This indicates that compared with perceived ease of use, the improvement of satisfaction, perceived usefulness, and perceived teacher support and self-efficacy services of mobile learning can more significantly enhance students' willingness to continue using mobile learning. In addition, the path analysis shows that perceived teacher support has a greater impact on satisfaction and self-efficacy on perceived usefulness. Therefore, the service focusing on improving perceived teacher support and self-efficacy can not only improve perceived usefulness and satisfaction, but also promote the increase of students' willingness to continue using $\mathrm{m}$ learning. This also indicates that the behavior and attitude of teachers' supporting students and the enthusiasm and confidence of students in mobile learning should be paid attention to when improving and enhancing the willingness to continue using mobile learning.

This study introduces student behavior and teacher support behavior to study the willingness to continue using mobile learning, which proves the research model constructed in this paper, but it still has certain limitations. Although some of the data in this paper have reached acceptable standards, the degree of fitting needs to be improved. At present, the common methods to improve the degree of fit include expanding or reducing the sample size, replacing the sample, changing the sample model, and adding model explanatory variables. In the future, this paper will further optimize the model variables to improve the degree of fit based on the current research data and reference materials. In addition, with the continuous development and segmentation of mobile learning, there will be influencing factors not included in this paper. Future research can try different theories, add different variables and other methods to build a more complete model.

\section{References}

1. Jongpil Cheon, Sangno Lee, Steven M. Crooks, Jaeki Song. An investigation of mobile learning readiness in higher education based on the theory of planned behavior[J].Computers \& education, 2012,59(3): 1054-1064.

2. ARK S Y,NAM M W,CHA S B, University students, behavioral intention to use mobile learning: evaluating the technology acceptance model[J].British journal of educational technology,2011,43(4):592-605.

3. Altihunibat, A. Determining the factors influencing students intention to use $\mathrm{m}$ learning in Jordan higher education [J].computers in Human Behavior.2015(52):65-71 
4. Mahat,J.,Ayub, A.F.M., Luan , S.,\& Wong.(2012). An assessment of students' mobile self-efficacy, readiness and personal innovativeness towards mobile learning in higher education in Malaysia[J]. Procedia-Social and Behavioral Sciences, (64): 284-290.

5. Ryan,R.M.\&E.L.Deci.2000.Intrinsic and extrinsic motivations: Classic definitions and new directions. Contemporary Educational Psychology 25:54-67.

6. Bhattacherjee, A. Understanding Information Systems Continuance: An Expectation Confirmation Model [J]. MIS Quarterly.2001,(25:3), pp. 351-370. 